\title{
Klassifizierung der Mammachirurgie
}

\section{J. Hoffmann, D. Wallwiener}

Eine der Grundvoraussetzungen, um evidenzbasierte Daten zu operativen Verfahren zu generieren, ist die Verfügbarkeit von Klassifizierungssystemen. Versuche, Brustkrebsoperationen zu klassifizieren, haben bisher immer mehr auf bestimmte Probleme fokussiert, z.B. die Vermeidung sekundärer chirurgischer Eingriffe, und weniger auf eine übergreifende Strukturierung. Ausgehend von einem existierenden Schema, das in der Universitäts-Frauenklinik Tübingen zur Standardisierung von OP-Berichten eingesetzt wird, wurde eine neue Klassifikation für ablative und brusterhaltende OP-Verfahren entwickelt und systematisch ausgearbeitet. Um diese neue Klassifikation zu überprüfen, wurde eine prospektive Analyse von Patientenberichten für alle größeren Eingriffe an der Brust im Brustzentrum der UniversitätsFrauenklinik Tübingen zwischen 2005 und 2005 durchgeführt, wobei die neue Klassifikation genutzt wurde [1].

Das neue System unterscheidet zwei Typen und sechs Klassifikationen, aufgeteilt in 12 Hauptkategorien, 13 Subkategorien und 39 Sub-Subkategorien von onkologischen, onkolplastischen und rekonstruktiven Eingriffen an der Brust (siehe Tab. 1) [2]. Das System gestattete eine einheitliche Klassifikation, aller 1225 Operationen bei 1166 Brustkrebspatientinnen in den Jahren 2005 und 2006. Ausnahmen oder weitere Unterteilungen waren nicht notwendig.

Onkochirurgische, onkoplastische und rekonstruktive Eingriffe an der Brust können grundsätzlich entsprechend ihrer chirurgi- schen Komplexität klassifiziert werden. Eine Analyse aller am Brustzentrum der Universitäts-Frauenklinik Tübingen durchgeführten Eingriffe bestätigte, dass die hier entwickelte neue Klassifikation ohne Ausnahme für alle diese Eingriffe angewendet werden konnte.

\section{Literatur}

1 Hoffmann J, Wallwiener D. Classifying breast cancer surgery: a novel, complexity-based system for oncological, oncoplastic and reconstructive procedures, and proof of principle by analysis of 1225 operations in 1166 patients. BMC Cancer 2009; 9: 108

2 Wallwiener D et al. Qualitätssicherung in der Frauenheilkunde. S. Kramarz; 2010: $170 \mathrm{ff}$.

\section{Bibliografie}

DOI http://dx.doi.org/10.1055/s-0031-1271515

Senologie 2011; 8: 99

(c) Georg Thieme Verlag KG Stuttgart · New York · ISSN 1611-6453

\section{Korrespondenzadresse}

\section{Dr. med. Jürgen Hoffmann}

Universitäts-Frauenklinik Tübingen

Calwerstr. 7

72076 Tübingen

j.hoffmann@med.uni-tuebingen.de

Tab.1 Klassifikation ablativer und brusterhaltender Operationsverfahren beim Mammakarzinom unter onkologischen, onkoplastischen und rekonstruktiven Gesichtspunkten nach Komplexitätsgraden 1 bis 6 (nach Hoffmann J, Wallwiener D, BMC Cancer 2009 [2]).

\begin{tabular}{|c|c|c|c|}
\hline \multirow{4}{*}{ onkologisch } & & Mammakarzinom-Operationen & \\
\hline & $\begin{array}{l}\text { Komplexitäts- } \\
\text { grad }\end{array}$ & $\begin{array}{l}\text { ablative Operation } \\
\text { des Mammakarzinoms }\end{array}$ & $\begin{array}{l}\text { brusterhaltende Operation } \\
\text { des Mammakarzinoms }\end{array}$ \\
\hline & 1 & $\begin{array}{l}\text { einfache (modifiziert radikale) Exzision } \\
\text { ohne Rekonstruktion }\end{array}$ & $\begin{array}{l}\text { einfache Exzision (direkte Adaptation) bis zur Qua- } \\
\text { drantektomie, Defektdeckung ohne Mobilisationen }\end{array}$ \\
\hline & 2 & $\begin{array}{l}\text { komplexe (radikale) Mastektomie } \\
\text { ohne Rekonstruktion }\end{array}$ & $\begin{array}{l}\text { komplexe Operation plus intramammäre Drüsen- } \\
\text { körper-Rekonstruktion, kleinflächige Mobilisationen, } \\
\text { Defektdeckung durch Mobilisation } \leq \mathbf{2 5} \%\end{array}$ \\
\hline \multirow{4}{*}{$\begin{array}{l}\text { onkoplastisch } \\
\text { (primär } \\
\text { rekonstruktiv) } \\
\text { oder } \\
\text { (sekundär) } \\
\text { rekonstruktiv }\end{array}$} & 3 & $\begin{array}{l}\text { onkoplastische oder rekonstruktive } \\
\text { Mammakarzinom-Operation } \\
\text { mit Prothesenrekonstruktion }\end{array}$ & $\begin{array}{l}\text { onkoplastische bzw. rekonstruktive Mammakarzinom- } \\
\text { Operation mit Hautresektion, Rezentrierung, glanduläre } \\
\text { Lappenplastiken, weitflächige Mobilisation. } \\
\text { Defektdeckung durch Mobilisation }>25 \%\end{array}$ \\
\hline & 4 & $\begin{array}{l}\text { komplexe onkoplastische oder rekon- } \\
\text { struktive Mammakarzinom-Operation } \\
\text { mit lokaler Lappenplastik }\end{array}$ & $\begin{array}{l}\text { tumoradaptierte Lifting-Operationen mit komplexen } \\
\text { Umschneidungsfiguren - tumoradaptierte } \\
\text { Mastopexie - oder Tumorresektion mit } \\
\text { Defektdeckung über lokale Lappenplastik }\end{array}$ \\
\hline & 5 & $\begin{array}{l}\text { komplexe onkoplastische oder rekon- } \\
\text { struktive Mammakarzinom-Operation } \\
\text { mit gestielter Fernlappenplastik: } \\
\text { LDF, TRAM-Flap }\end{array}$ & $\begin{array}{l}\text { komplexe onkoplastische bzw. rekonstruktive } \\
\text { Mammakarzinom-Operation mit zusätzlicher Resektion } \\
\text { (Reduktion) von Brustdrüsengewebe - } \\
\text { tumoradaptierte Reduktionsplastik }\end{array}$ \\
\hline & 6 & $\begin{array}{l}\text { komplexe onkoplastische bzw. rekonstruk- } \\
\text { tive Mammakarzinom-Operation mit } \\
\text { freier Lappenplastik und mikrovaskulärem } \\
\text { Gefäßverschluss: } \\
\text { DIEP, SIEA, SGAP, free-TRAM }\end{array}$ & $\begin{array}{l}\text { komplexe onkoplastische bzw. rekonstruktive Mamma- } \\
\text { karzinom-Operation mit Defektdeckung über gestielte } \\
\text { (LDF, TRAM-Flap) oder freie (z. B. DIEP, SIEA, SGAP) } \\
\text { Fernlappenplastiken } \\
\text { ggf. mit mikrovaskulärem Gefäßanschluss }\end{array}$ \\
\hline
\end{tabular}

